\title{
ESTIMATION OF RAINFALL RATE IN EASTERN TIBET USING GROUND-BASED RADAR OBSERVATIONS: METHOD DEVELOPMENT
}

\author{
Petra KOUDELOVA ${ }^{1}$ and Toshio KOIKE ${ }^{2}$ \\ ${ }^{1}$ Member of JSCE, Ph.D., Japan Science and Technology Corporation, Dept. of Civil Engineering, University of \\ Tokyo (7-3-1 Hongo, Bunkyo-ku, Tokyo 113, Japan) \\ ${ }^{2}$ Member of JSCE, Professor, Dept. of Civil Engineering, University of Tokyo (7-3-1 Hongo, Bunkyo-ku, Tokyo
} 113, Japan)

\begin{abstract}
This paper proposes a method for estimating rainfall rate from ground-based radar observations in eastern Tibet. Firstly, the radar reflectivity - rain rate $(Z-R)$ relationship is derived by regression analysis using the hourly data of a single rain gauge located near the radar and the corresponding radar reflectivity over a period of more than two months. Two different relationships are obtained for stratiform and convective rain types. Secondly, an automated convective-stratiform rain classification method is introduced that uses the horizontal radar echo structure to identify convective regions and classifies the remainder as stratiform areas. The classification algorithm is verified against visual inspections of horizontal and vertical radar echo structures. Finally, the rain rate is estimated from the radar reflectivity for the rain gauge site using the derived $Z-R$ relationships and the rain type information produced by the classification algorithm. The comparison of the estimation and the gauge data suggests good accuracy.
\end{abstract}

Key Words : GAME-Tibet, radar reflectivity - rainfall rate relation, radar echo classification

\section{INTRODUCTION}

The Global Energy and Water Cycle Experiment (GEWEX) Asian Monsoon Experiment (GAME)-Tibet project was conducted to clarify the land surface-atmosphere interactions over the Tibetan Plateau in the context of the Asian monsoon system $^{1)}$. In GAME-Tibet, extensive field works were conducted in the Naqu basin in eastern Tibet (known as the meso-scale experimental area) in September 1997 for the Preliminary Observation Period (POP) and from May to September 1998 for the Intensive Observation Period (IOP $)^{2)}$.

One of the key issues of GAME-Tibet is the hydrological analysis of land surface processes by employing a distributed hydrological model coupled with a land surface scheme. For such a study, the quantitative evaluation of spatially- and temporallydistributed rainfall is of fundamental importance. A precipitation gauge network (PGN) ${ }^{3)}$ was installed during GAME-Tibet in order to provide hourly measurements of rainfall and snowfall over the meso-scale experimental area. Rain gauges measure rainfall directly, but only at the location of the instrument, and thus they do not provide information on the distribution of rain with sufficient resolution for the intended hydrological analysis ${ }^{4)}$. The X-band Doppler radar of the National Space Development Agency of Japan (NASDA) was deployed during GAME-Tibet to provide radar reflectivity data of high spatial resolution in order to be able to obtain rain distribution patterns and areal rainfall amounts over the area of interest ${ }^{5)}$. Radar reflectivity (echo) $Z$ is related physically (through Rayleigh scattering theory) to rainfall rate $R$ via the raindrop size distribution ${ }^{6}$. However, there are many uncertainties in estimating rain rate using radar reflectivity (e.g., the distant position of the radar beam above the surface, anomalous propagation echoes, the natural variability in the rain drop size distribution, various issues to do with the beam geometry, attenuation of the echo, and random error in the reflectivity measurement, etc.). Therefore, the only viable way to quantitatively evaluate rain is to combine radar and rain gauge information. The rain gauge data are used to calibrate the radar reflectivity in the vicinity of the gauge for identifying the radar 
reflectivity-rain rate $(Z-R)$ relationship.

The main motivation for the presented study is to provide an effective methodology which can be used for estimating the spatially-distributed rain rate using radar observations in the GAME-Tibet mesoscale experimental area. In this paper, we introduce and validate a method for a single observation site near the radar, namely the Naqu hydrological (NaquHY) station (Figure1). The possibility of expanding the method for the whole of the area of radar coverage is discussed in the following sections.

In the proposed method, we combine regression analysis, which is used to derive the $Z-R$ relationship, and a radar echo classification algorithm (hereinafter referred as RCA). The reason for including the RCA is the evident dependency of the $Z-R$ relation on whether the rainfall is convective or stratiform, found by analysing the observed data. Accordingly, two $Z-R$ relationships are derived using the NaquHY rain gauge data and the associated radar reflectivity records. For the NaquHY station, the radar echo type, which corresponds to the rain type, is classified by the visual inspection of the horizontal and vertical radar echo structures. In order to determine the rain type over a wider area and for each rain event over the examined period, an automated RCA is required. In this study, the algorithm proposed by Steiner et al. ${ }^{7)}$ is adopted. Based on the RCA results, the appropriate $Z-R$ relationship is selected and the rain rate is estimated at the NaquHY station over the examined period. The estimated rain rate is verified against rain gauge observations on hourly and daily time scales. The comparison suggests that the method provides satisfactory results.

\section{DATA}

Figure 1 shows a map of the instruments located in the GAME-Tibet meso-scale experimental area, which covers the Naqu basin. The NASDA X-band Doppler radar was set up on the hill $15 \mathrm{~km}$ southwest of Naqu city $\left(31.4^{\circ} \mathrm{N}, 91.9^{\circ} \mathrm{E}, 4584 \mathrm{~m}\right.$ ASL). The radar was operated continuously over the POP and IOP at a 10-minute temporal resolution ${ }^{8)}$. However, the radar system sometimes stopped due to mechanical problems and hence the records are not fully continuous. Moreover, adjustments on the system were done at the end of August and thus the September records are not considered in this study. The 3-D orthogonal gridded data of radar reflectivity were constructed from the radar observations. This study uses 2-D horizontal reflectivity data at a height of $1.5 \mathrm{~km}$, which covers a circle of the radius of $240 \mathrm{~km}$ with a grid size of 2

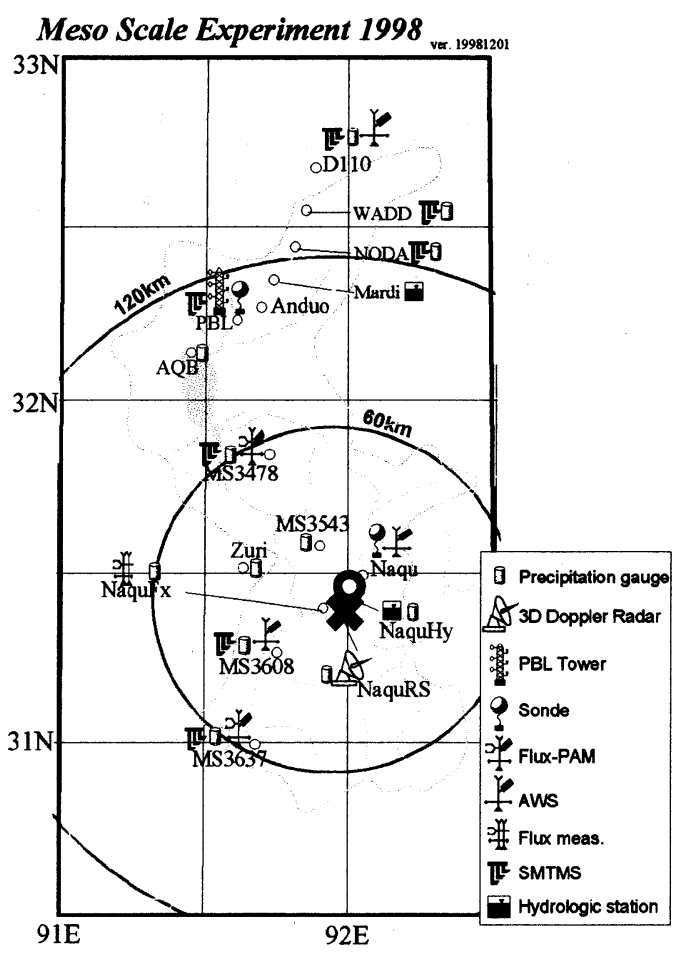

Fig.1 Map of the GAME-Tibet mesoscale experiment. The $\boldsymbol{*}$ and $\boldsymbol{O}$ symbols indicate radar site and NaquHY station with DFSG, respectively.

$\mathrm{km}$.

Due to its parameters, the X-band radar used (see e.g. ${ }^{8)}$ ) is subject to attenuation by hydrometeors, which affects the observed reflectivity and thus is an important factor in the $Z-R$ relationship. In order to avoid the effects of attenuation in this study, we selected a rain gauge located near the radar site where these effects can be neglected. The selected site is located near Naqu city (NaquHY station) ${ }^{3)}$ at $31.42^{\circ} \mathrm{N}, 91.98^{\circ} \mathrm{E}, 4493 \mathrm{~m} \mathrm{ASL}$, which is about 7 $\mathrm{km}$ northeast of the radar site. At this site, a double-fenced standard rain gauge (DFSG) was established. The double fence reduces the effects of wind on precipitation measurements and thus the observations of DFSG are considered to be reliable. However, an attenuation correction of the radar reflectivity should be made before application of the proposed method to the whole of the area of the radar coverage.

The data are available through the homepage of GAME-Tibet: http://monsoon.t.u-tokyo.ac.jp/tibet.

\section{RADAR REFLECTIVITY - RAIN RATE RELATIONSHIP}

Both the radar reflectivity $Z$ and the rain rate $R$ are different functions of the rain drop size distribution (DSD). Using the definitions of $Z$ and $R$ in the terms of the DSD, Uijlenhoet ${ }^{6)}$ demonstrated that these definitions naturally lead to a power law $Z-R$ relationship: 


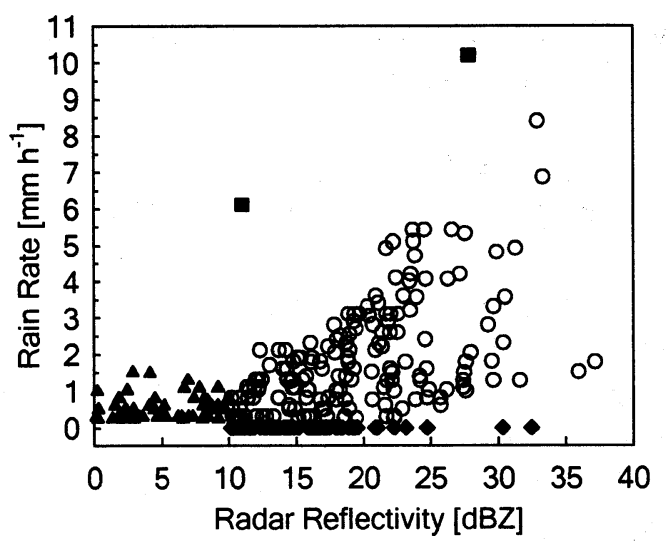

Fig.2 Rain rate at the DFSG versus the corresponding radar reflectivity. Open circles: ensemble $\mathrm{A}$, full diamond: ensemble $\mathrm{B}$, full triangles: ensemble $\mathrm{C}$, full squares: unreliable extremes; explained in the text.

$$
Z=\alpha \cdot R^{\beta},
$$

where $Z$ is the equivalent radar reflectivity factor $\left(\mathrm{mm}^{6} \mathrm{~m}^{-3}\right), R$ is the rainfall intensity $\left(\mathrm{mm} \mathrm{h}^{-1}\right)$, and $\alpha$ and $\beta$ are positive coefficients, which are related to the parameters of the DSD. Thus, the coefficients $\alpha$ and $\beta$ can be determined from measured or estimated DSD in a given precipitation regime ${ }^{9), 10)}$.

In this study, we derive the $Z-R$ relationship directly by regression analysis using the DFSG data and the radar reflectivity data for the pixel $(2 \times 2 \mathrm{~km})$ in which the DFSG is located. One of the reasons for this is the lack of sufficient data for carrying out methods utilizing the DSD. Moreover, the relationship derived by the regression incorporates many of the problems associated with radar and rain gauge measurements and with the rain type. On the other hand, the derived relationship is valid locally for in the vicinity of the DFSG and should be calibrated using other rain gauges before applying this method to the whole of the area of radar coverage.

Since radar reflectivity $Z$ varies over a wide range of values from units to dozens of thousands, it is often converted to decibel units of radar reflectivity with $d B Z\left(=10 \cdot \log _{10} Z\right)$. Using this logarithmic expression, we can rewrite Eq. (1) as:

$$
R=a \cdot b^{d B Z},
$$

where $a$ and $b$ are empirical coefficients to be determined by regression analysis. The regression analysis is performed on an hourly basis, which corresponds to the temporal resolution of the DFSG records and also the temporal resolution of the spatially-distributed rainfall estimates required for hydrological studies. The 10-minutes records of reflectivity $Z$ are averaged over an hour and then converted to $d B Z$ to obtain a consistent dataset for the regression analysis.

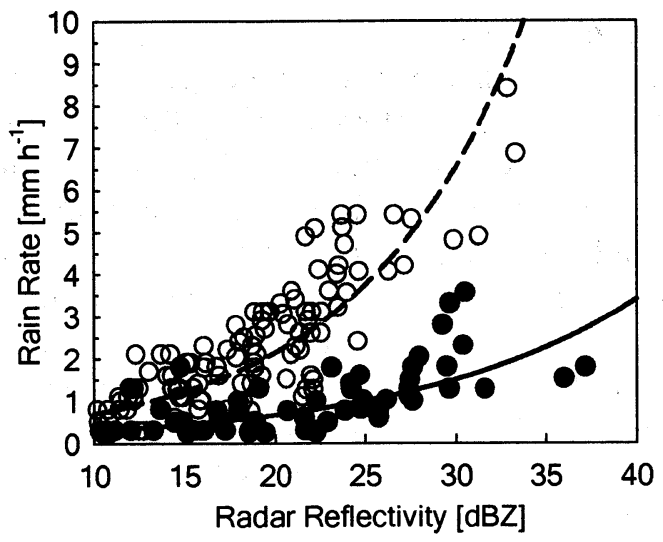

Fig.3 The matching data of ensemble A sorted according to the rain type. Open circles: stratiform, full circles: convective. The obtained regression curves are depicted by the dashed line for stratiform and solid line for convective type.

A comparison of the corresponding pairs of reflectivity $(d B Z)$ and rain rate is shown in Figure 2. The cases in which zero rainfall corresponds to reflectivities of lower than the threshold value indicating rain $(10 \mathrm{dBZ})^{9), 11)}$ are excluded. The chart reveals that in some cases the reflectivity and the rain rate do not match, i.e. either zero rain corresponds to a reflectivity of $\geq 10 \mathrm{dBZ}$ (ensemble $\mathrm{B})$, or a nonzero rain corresponds to a reflectivity of $<10 \mathrm{dBZ}$ (ensemble C). The mismatched data are irrelevant in terms of the regression and thus they are excluded in the further analysis, as are the two unreliable extremes, which are inconsistent with the rest of the data.

As a result, we have 152 pairs of matching data (hereinafter referred as ensemble A - indicated by open circles in Figure 2) for the regression procedure. However, even the matching data are quite scattered. Regarding this scatter, the ensemble A will be further examined from the point of view of cloud characteristics. Basically, there are two types of precipitation in Tibet during summer. During the daytime, the occurrence of local convective precipitation of weak intensity is quite common. Stratiform precipitation prevails during the nighttime and is usually stronger than the convective precipitation ${ }^{3)}$. The horizontal and vertical radar echo structure of stratiform and convective rain differs remarkably. The typical features of the convective echo are a strong horizontal reflectivity gradient, a weak vertical reflectivity gradient due to the convective mixing, and usually only local occurrence. On the other hand, the stratiform echo is usually spread over a wider area, the horizontal reflectivity gradient is weak, and the echo is vertically stratified. If the stratiform cloud is well developed, a bright band of intense echo is evident in the vertical profile of the reflectivity, corresponding to the melting layer of 
the stratiform cloud.

Accordingly, the horizontal images of the radar echo for each rain event recorded by the DFSG were visually inspected and the echo type at the site of DFSG was identified, if it was clear from the horizontal structure. Otherwise, the vertical cross-sections in the west-east and north-south directions were also examined. Consequently, the ensemble A data were sorted (Figure 3), suggesting a different $Z-R$ relationship for each rain type. The respective regression curves were obtained for stratiform rain as:

$$
R=0.23 \cdot 1.118^{d B Z}
$$

and for convective rain as:

$$
R=0.13 \cdot 1.085^{d B Z} \text {. }
$$

The root mean square errors (RMSE) and mean bias errors (MBE) were calculated by:

$$
\begin{aligned}
& R M S E=\sqrt{\frac{\sum(P-O)^{2}}{N},} \\
& M B E=\frac{\sum(P-O)}{N},
\end{aligned}
$$

where $P$ is the predicted rain rate by the derived relationships, $O$ is the observed, and $N$ is the number of data pairs. The errors are listed in Table 1. The RMSE and the MBE are of the order of tenths of $\mathrm{mm} \mathrm{h}^{-1}$ for both the convective and the stratiform relationships, showing a sufficient accuracy of prediction. The RMSE of the stratiform relationship is higher than the RMSE of the convective one, indicating that a bigger error in rain rate estimation can be expected for the stratiform rainfall. The bias errors signal a small underestimation in the rain rate by both the convective and the stratiform relationships.

Table 1 The RMSE and the MBE of the regression relationships (3) and (4). The errors are calculated considering the matched data only.

\begin{tabular}{|c|c|c|}
\hline Rain type (N) & RMSE $\left(\mathrm{mm} \mathrm{h}^{-1}\right)$ & MBE $\left(\mathrm{mm} \mathrm{h}^{-1}\right)$ \\
\hline Convective (39) & 0.58 & -0.12 \\
\hline Stratiform (113) & 0.85 & -0.15 \\
\hline
\end{tabular}

The mutual relationship between the stratiform and the convective relationships is very interesting. Usually, the same reflectivity corresponds to a higher rate in the case of convective rain and a lower rate in the case of stratiform rain, while for Tibet, we obtained the opposite relationship. This unusual feature is perhaps caused by the fact that the melting layer of the stratiform clouds occurs at a relatively low elevation above the surface due to the high altitude of the Tibetan Plateau. The radar beam thus looks at the snow particles, which are characterized by lower reflectivities than for raindrops at the ground.

\section{CONVECTIVE-STRATIFORM ECHO CLASSIFICATION ALGORITHM}

The application of the derived relationships (3) and (4) is conditional on knowledge of the radar echo type. The visual inspection of the radar echo, which could be carried out for a single pixel, is impracticable for areal data of long-term observations, and hence an automated radar echo classification algorithm (RCA) is needed.

Several approaches have been taken attempting to classify radar echoes. Some methods use the radar reflectivity bright band (BB) to identify stratiform echoes. Since the BB typically has a vertical extent of a few hundreds of meters, this approach has a severe limitation for ground-based radars because of the coarse vertical resolution due to the widening of the radar beam with distance from the radar ${ }^{7}$. Moreover, the $0^{\circ} \mathrm{C}$ level occurs very near the surface in Tibet (around $700-1000 \mathrm{~m}$ above the surface) and thus the BB may be completely missed or appears in the lowest level of the vertical reflectivity profile, which makes its identification uncertain, especially for farther ranges of the radar coverage (e.g., embedded convection can be mistaken for a BB at coarse vertical resolution).

Therefore, we have adopted the method of Steiner et al. ${ }^{7}$, which utilizes the horizontal structure of the radar echo. The basic idea is to search for peaks in reflectivity and to determine the radius of the convective region. All of the pixels within this region are classified as convective and the remainder is classified as stratiform. The criteria for identifying convective echoes are:

1) Intensity: Any pixel with a reflectivity of at least $40 \mathrm{dBZ}$ is tagged as a convective core, since stratiform echoes cannot practically be so high.

2) Peakedness: Any pixel not identified as a convective core in the first step, but with a reflectivity exceeding the average value taken over the surrounding background by at least a certain reflectivity difference $\Delta Z$ is also identified as a convective core. The background reflectivity is determined as the linear average of the nonzero echoes $\left(Z\right.$ in $\left.\mathrm{mm}^{6} \mathrm{~m}^{-3}\right)$ within a radius of $11 \mathrm{~km}$ around the pixel and then converted into $\mathrm{dBZ}$. The reflectivity difference by which the radar echo must exceed the background value to become identified as a convective core is given by:

$$
\Delta Z=\operatorname{MAX}\left(0,10-Z_{b s}^{2} / 160\right),
$$

where $\Delta Z$ is in decibels and $Z_{b g}$ is the background reflectivity in $\mathrm{dBZ}$. Eq. (7) has been tuned for the 
GAME-Tibet IOP radar data following the method of Steiner et al. ${ }^{7}$.

3) Surrounding area: For each pixel identified as a convective core, all of the surrounding pixels within an intensity-dependent convective radius around that pixel are also classified as being part of the convective area. The convective radius is given by:

$$
d_{c}=\operatorname{MIN}\left(5, \operatorname{MAX}\left(1, \operatorname{INT}\left(\left(Z_{b g}-15\right) / 5\right)\right)\right),
$$

where $d_{c}$ is the convective radius in $\operatorname{km}$ and INT $(x)$ indicates the conversion of argument $x$ to an integer by truncation.

\section{VALIDATION}

In this section, the methodology for the estimation of rain rate using the radar reflectivity observations described in Sections 3 and 4 is validated for the DFSG site.

Firstly, the RCA was applied to the 2-D GAME-Tibet IOP radar reflectivity data with a 10-min. temporal resolution. The results are averaged for an hourly classification so that the prevailing echo type within a particular hour is prescribed as a characteristic type for that hour. If the frequency of convective and stratiform echoes within a particular hour is the same, a stratiform echo is identified during the nighttime (20:00 06:00 local time) and a convective echo during the daytime (07:00 - 19:00 local time) in accordance with the findings of Ueno et al. ${ }^{3)}$. The results of the RCA for the DFSG site correspond well with the visual inspection of the radar echo structure. The agreement in echo type identification was in $82 \%$ of all of the data of the ensemble A.

The rain rate was then estimated for the DFSG site using the derived $Z-R$ relationships (3) and (4) according to the classification by the RCA. Thus, the estimation includes both the error introduced by the RCA and the error in the $Z-R$ relationship. The estimated rain rates are verified against the observation of the DFSG in Figure 4a. The circles in the chart are located closely along the identity line, which suggests a good accuracy of the method. The higher density of the circles above the identity line indicates a negative bias (underestimation).

The results were also examined on a daily time-scale basis. The daily totals of rain estimates were compared with the daily totals of the observed rain rates (Figure 4b). Again, a relatively good agreement is indicated. Compared to the hourly time-scale analysis, the number of mismatched data points (ensemble B and C in Section 3) decreased. This suggests that some mismatched pairs in the hourly time-scale ensemble may have occurred as a a)

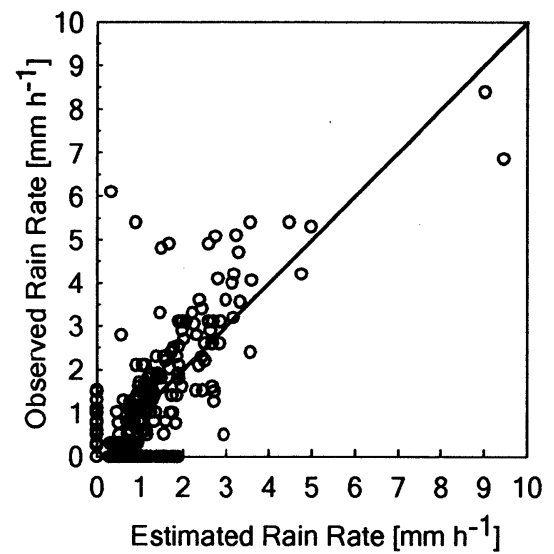

b)

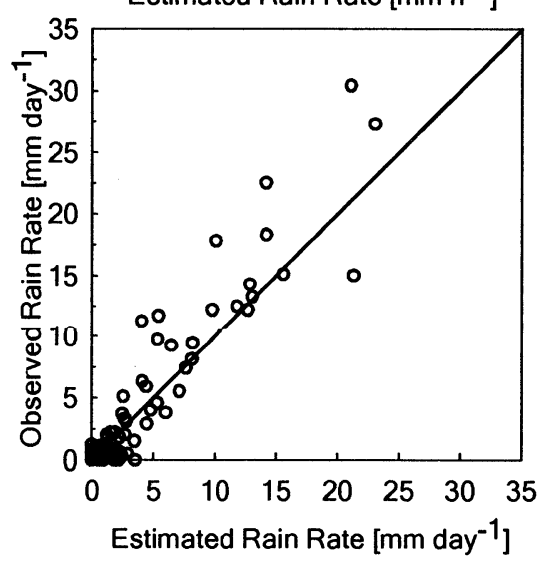

Fig.4 Relations of a) estimated and observed hourly rain rate and b) daily totals of estimated and observed rainfall for the DFSG site over the examined period. The solid line denotes identity.

result of slightly different timing of radar and gauge observations, which is removed by integrating over a day.

Table 2 The RMSE, the MBE (both in $\mathrm{mm} \mathrm{h}^{-1}$ or $\mathrm{mm} \mathrm{day}^{-1}$ ), and the $\mathrm{CC}$ of the rain estimation method for the hourly and daily time scales, respectively.

\begin{tabular}{|c|c|c|c|}
\hline Ensemble (N) & RMSE & MBE & CC \\
\hline Hourly (152) & 1.1 & -0.286 & 0.758 \\
\hline Daily (52) & 3.0 & -0.83 & 0.924 \\
\hline
\end{tabular}

The RMSE, the MBE, and the correlation coefficient (CC) were calculated for both hourly and daily time scales in order to evaluate the accuracy of the proposed methodology. The RMSE and the $\mathrm{MBE}$ are given by Eqs. (5) and (6), and the $\mathrm{CC}$ was calculated by:

$$
C C=\frac{N \sum P O-\sum P \sum O}{\sqrt{\left(N \sum P^{2}-\left(\sum P\right)^{2}\right)\left(N \sum O^{2}-\left(\sum O\right)^{2}\right)}}
$$

The errors and the $\mathrm{CC}$ were calculated considering only the matched data and are listed in Table 2.

The hourly time-scale RMSE and MBE are higher than the errors of the regression $Z-R$ relationship identified in Section 3. This error increment is introduced by the RCA due to the false identification of the echo type. The error increment 
is obvious, especially in the case of the MBE, in which the signal again systematically underestimates the rainfall. Since the stratiform rain is usually stronger than the convective rain in the Naqu basin, the increment of the negative bias indicates that cases when stratiform rain is falsely identified as convective occur more often than the opposite false classification (convective classified as stratiform). This is consistent with the fact that stratiform rain occurred much more often during the examination period. The $\mathrm{CC}$ is relatively high, which suggests a good agreement with the observations. Also, the low absolute values of the errors signal sufficient accuracy in the estimation.

The daily time-scale RMSE and MBE exhibit similar patterns as the hourly time-scale errors. The $\mathrm{CC}$ is higher, which suggests that the accuracy increases when the results are integrated over a longer period. This tendency is confirmed by comparing the totals of the estimated rainfall $\left(R e_{t o t}=\right.$ $317 \mathrm{~mm})$ and the observed rainfall $\left(R o_{t o t}=356 \mathrm{~mm}\right)$ over the whole of the period examined. These total values indicate that the error in the long-term rainfall accumulation estimation is about $11 \%$.

\section{CONCLUSIONS}

This paper presented the methodology for the estimation of rain rate in the GAME-Tibet meso-scale experiment area using ground-based radar observations. The technique was developed for a single site near the radar with the intention that it could be extended to the whole of the area of the radar range.

The method included the derivation of the $Z-R$ relationship from radar and reference rain gauge data over a period of more than 2 months (June August). Two different relationships were obtained by regression analysis, referring to convective and stratiform rain types.

Since the application of the $Z-R$ relationship is conditional on the knowledge of the rain type, an automated radar echo classification algorithm was introduced to make the methodology applicable for large areal datasets. The algorithm is based on the intensity and sharpness of the maxima in the horizontal patterns of reflectivity.

The methodology was tested for the reference gauge site. The classification algorithm was run for the whole of the period examined. Following the classification procedure, the $Z-R$ relationship corresponding to the rain type was used to estimate the rain rate. The rain estimates calculated in that way were compared with the observation of the reference rain gauge for the hourly and daily time scales. The comparison indicated that the method is sufficiently accurate. Therefore, we suggest that the proposed method may be applicable to the whole of the area of the GAME-Tibet radar coverage by introducing further information, especially the radar attenuation correction and calibration using other rain gauges within the area.

ACKNOWLEDGMENT: The data used in this paper were obtained through the GAME-Tibet project supported by the Ministry of Education, Science, Sports and Culture of Japan; the Science and Technology Agency of Japan, the Chinese Academy of Science; the Frontier Research for Global Change; the National Space Development Agency of Japan; and the Asian Pacific Network.

\section{REFERENCES}

1) GAME International Science Panel: GEWEX Asian Monsoon Experiment (GAME) implementation Plan, 1998.

2) Koike, T., Yasunari, T., Wang, J. and Yao, T.: GAME-Tibet IOP Summary Report, Proc. of the $1^{\text {st }}$ Int. Work. on GAME-Tibet, Xi'an, China, pp.1-2, 1999.

3) Ueno, K., Fujii, H., Yamada, H. and Liu, L.: Weak and frequent monsoon precipitation over the Tibetan Plateau, $J$. Meteor. Soc. Japan, Vol.79, No.1B, pp.419-434, 2001.

4) Wood, S. J., Jones, D. A. and Moore, R. J.: Accuracy of rainfall measurement for scales of hydrological interest, Hydrol. Earth Syst. Sci., Vol.4, pp.531-541, 2000.

5) Shimizu, S., Ueno, K., Fujii, H., Yamada, H., Shirooka, R. and Liu, L.: Summary of Doppler radar observation on the Tibetan Plateau and the preliminary results, Proc. of the $I^{\text {st }}$ Int. W. on GAME-Tibet, Xi'an, China, pp.113-116, 1999.

6) Uijlenhoet, R.: Raindrop size distributions and radar reflectivity - rain rate relationships for radar hydrology, Hydrol. and Earth Syst. Sci., Vol.5(4), pp.615-627, 2001.

7) Steiner, M., Houze R. A. and Yuter S. E.: Climatological characterization of three-dimensional storm structure from operational radar and rain gauge data, J. Appl. Meteor., Vol.34, pp.1978-2007, 1995

8) Shimizu, S., Ueno, K., Fujii, H., Yamada, H., Shirooka, R. and Liu, L.: Mesoscale characteristics and structures of stratiform precipitation on the Tibetan Plateau, J. Meteor. Soc. Japan, Vol.79, No.1B, pp.435-461, 2001.

9) Yuter, S. E. and Houze, R. A.: Measurements of raindrop size distributions over the pacific warm pool and implications for Z-R relations, J. Appl. Meteor., Vol.36, pp.847-867, 1997.

10) Nakagawa, K., Nakakita, E., Ikebuchi, S., Sato, T., Takasao, T.: Formulation of vertical profile of rain drop size distribution, Proc. of the Int. Conf. on Water Res. \& Env. Res.: Towards the $21^{\text {st }}$ century, Kyoto, Japan, Vol.1, pp.651-658, 1996.

11) Uyeda, H., Yamada, H., Horikomi, J., Shirooka, R., Liu, L., Ueno, K., Fujii, H. and Koike, T.: Characteristics of convective clouds observed by a Doppler radar at Naqu on Tibetan Plateau during the GAME-Tibet IOP, J. Meteor. Soc. Japan, Vol.79, No.1B, pp.463-474, 2001.

(Received September 30, 2003) 